\title{
Trichosporon dohaense, a rare pathogen of human invasive infections, and literature review
}

This article was published in the following Dove Press journal: Infection and Drug Resistance

\begin{abstract}
Shu-Ying Yu, ${ }^{1-3} \mathrm{Li}-\mathrm{Na}$ Guo, ${ }^{1,3}$
Meng Xiao, ',3 Timothy

Kudinha, ${ }^{4,5}$ Fanrong Kong, ${ }^{4} \mathrm{He}$ Wang, ${ }^{1,3}$ Jing-Wei Cheng, ${ }^{1-3}$ Meng-Lan Zhou, ${ }^{1-3}$ Hui Xu, ${ }^{6}$ Ying-Chun $\mathrm{Xu}^{\mathrm{l}-3}$

'Department of Clinical Laboratory, Peking Union Medical College Hospital, Chinese Academy of Medical Sciences, Beijing, China; ${ }^{2}$ Graduate School, Peking Union Medical College, Chinese Academy of Medical Sciences, Beijing, China; ${ }^{3}$ Beijing Key Laboratory for Mechanisms Research and Precision Diagnosis of Invasive Fungal Diseases, Beijing, China; ${ }^{4}$ Centre for Infectious Diseases and Microbiology Laboratory Services, ICPMR Pathology West, University of Sydney, Westmead Hospital, Westmead, NSW, Australia; ${ }^{5}$ Centre for Infectious Diseases and Microbiology Laboratory Services, Westmead Hospital, Westmead, NSW, Australia; ${ }^{6}$ Department of Clinical Laboratory, First Affiliated Hospital of Zhengzhou University, Zhengzhou, Henan, China
\end{abstract}

Correspondence: Ying-Chun Xu Department of Clinical Laboratory, Peking Union Medical College Hospital, Chinese Academy of Medical Sciences, No. I, Shuaifuyuan Wangfujing, Dongcheng District, Beijing I00730, China

Tel +86 I069159766

Fax +86 1069159766

Email xycpumch@139.com

Hui Xu

Department of Clinical Laboratory, The First Affiliated Hospital of Zhengzhou University, No. I, East Jianshe Road, Zhengzhou, Henan 450000, China Email xuhui8866@I26.com
Background: Trichosporon dohaense is a rare fungal species that has not been described in human invasive infections.

Patients and methods: In this study, we investigated two $T$. dohaense isolates from patients with invasive infections in two hospitals in China, as part of the China Hospital Invasive Fungal Surveillance Net (CHIF-NET) program. Both patients were under immunocompromised conditions. Results: On chromogenic agar, $T$. dohaense isolates were dark blue, similar to the color of Candida. tropicalis, but the characteristic moist colony appearance was quite different from that of T. asahii. The two isolates were misidentified as T. asahii and T. inkin by the VITEK 2 YST system. The rDNA internal transcribed spacer (ITS) region and the D1/D2 domain sequences of the two T. dohaense isolates were $100 \%$ identical to $T$. dohaense type strain $\mathrm{CBS}_{10761^{\mathrm{T}}}$. The sequence of the intergenic spacer region-1 also clearly distinguished the species. Of the three matrix-assisted laser desorption/ionization time-of-flight mass spectrometry systems, Bruker Biotyper and Autobio MS correctly identified the two isolates to species level, whereas Vitek MS systems misidentified them as T. ovoides or T. asteroides. Echinocandins exhibited no in vitro activities against the two $T$. dohaense isolates. In addition, the isolates exhibited intermediate susceptibility to fluconazole (with minimal inhibitory concentrations [MICs] of 8 and $16 \mu \mathrm{g} /$ $\mathrm{mL}$ ) and itraconazole, voriconazole, and posaconazole (MICs of $0.25-1 \mu \mathrm{g} / \mathrm{mL}$ ). T. dohaense demonstrated susceptibility to amphotericin B with MIC of $1 \mu \mathrm{g} / \mathrm{mL}$. The MICs of fluconazole and voriconazole in our study were higher than the $\mathrm{MIC}_{50}$ of 62 for T. asahii isolates ( 4 and 0.064 $\mu \mathrm{g} / \mathrm{mL}$ ) in the CHIF-NET program.

Conclusion: This case study points to a possible emergence of $T$. dohaense as an opportunistic human invasive fungal pathogen, and the reduced susceptibility should be noted.

Keywords: Trichosporon dohaense, invasive infection, emerging pathogen, identification, reduced susceptibility

\section{Introduction}

Trichosporon species are yeast-like fungi distributed widely in the environment; they commonly colonize the human skin and the respiratory or gastrointestinal tract but can cause lethal infections. ${ }^{1-5}$ Trichosporonosis basically occurs in immunocompromised hosts, with the infection most commonly associated with broad-spectrum antibiotic therapy, chemotherapy, organ transplantation, surgical operation, as well as long-term intravascular device implantation, especially in the setting of hematological malignancy patients. ${ }^{1-4,6}$ Furthermore, due to delayed diagnosis and the lack of an optimal treatment strategy, mortality due to trichosporonosis remains high $(50 \%-80 \%)$ in malignant hematological patients despite treatment with antifungal drugs. ${ }^{7,8}$ 
The development of modern molecular identification methods has helped greatly in the classification of the genus Trichosporon, and to date, 16 species considered potential causative agents of human infections have been described, including T. asahii, T. asteroides, T. cutaneum, T. inkin, T. japonicum, T. jirovecii, T. mucoides, T. dermatis, T. montevideense, T. coremiiforme, T. domesticum, T. faecale, T. loubieri, T. ovoides, T. dohaense, and T. mycotoxinivorans., ${ }^{4,7-11}$ Of these, $T$. dohaense was first described as causing superficial infections in immunocompromised patients in Qatar in $2009 .{ }^{6}$

Two cases of invasive infections caused by $T$. dohaense in two hospitals were identified under the China Hospital Invasive Fungal Surveillance Net (CHIF-NET) program. Here, we describe these two case studies, including the clinical and molecular characteristics of the two T. dohaense isolates, as well as their notably reduced susceptibility to azoles, especially fluconazole.

\section{Case review}

\section{Patient I}

A 56-year-old male, presenting with non-Hodgkin's lymphoma with rectal or bladder involvement, was admitted to the intensive care unit (ICU) of Peking Union Medical College Hospital, Beijing, China, on 30 September 2010. The patient suffered from acute renal failure and acute myocardial infarction. He received chemotherapy and hemodialysis daily from Day 1 through to Day 8 of admission, as well as broadspectrum antibiotics daily from Day 5 to Day 8, but he never had any exposure to antifungals during the hospitalization. He had a fever of up to $38.6^{\circ} \mathrm{C}$ on Day 5. A yeast isolate, identified as T. asahii by chromogenic agar (CHROMagar Candida; CHROMagar Company, Paris, France), was recovered on Day 11 from the blood culture taken on Day 7. The isolate was subsequently identified by rRNA gene sequencing as $T$. dohaense (see the following text). The patient was discharged against medical advice on Day 10 before the fungal isolate was identified and thus did not receive any antifungal therapy, although he was in a serious condition. The patient was subsequently lost to follow-up (Table 1).

\section{Patient 2}

An 81-year-old female presented to the First Affiliated Hospital of Zhengzhou University on 10 March 2013 for treatment of rectovaginal fistula and incisional hernia after abdominal fistulation; she had continuous abdominal pain, nausea, and vomiting. She received broad-spectrum antibiotics from Day 0 through to Day 38 of admission. She was transferred to the ICU, where she was inserted with a right subclavian vein catheter and intrathoracic drain on Day 8 and received red blood cell transfusion. Blood $(1,3)-\beta$-D-glucan level was significantly raised on Day 25. The patient had a fever of up to $39.2^{\circ} \mathrm{C}$ on Day 29 . Intravenous fluconazole was started

Table I Clinical features of two patients with invasive infection caused by Trichosporon dohaense

\begin{tabular}{|c|c|c|}
\hline Clinical feature & Patient I & Patient 2 \\
\hline Isolate ID number & IOPUI93 & $12 Z Z 130$ \\
\hline$\overline{\text { Age }}$ & 56 & 81 \\
\hline Gender & Male & Female \\
\hline Reason for hospital admission & Non-Hodgkin's lymphoma & Colorectal cancer \\
\hline Underlying disease & Acute renal failure, acute myocardial infarction & Hypertension, coronary heart disease, diabetes \\
\hline First isolation of $T$. dohaense $\left(\right.$ date $\left.^{\mathrm{a}}\right)$ & $6 / 10 / 2010(7)$ & $17 / 04 / 2013(37)$ \\
\hline \multicolumn{3}{|l|}{ Clinical status at time of positive culture } \\
\hline Immunosuppressive state & Yes & Yes \\
\hline Neutropenia $\left(<10^{9} / \mathrm{L}\right)$ & No & No \\
\hline Presence of CVC & No & Yes \\
\hline Broad-spectrum antibiotics & Yes & Yes \\
\hline Parenteral nutrition & No & Yes \\
\hline Surgery within 30 days & No & Yes \\
\hline Intensive care & Yes & Yes \\
\hline Previous antifungal agents within 30 days & No & Yes \\
\hline Indwelling urinary catheter & Yes & No \\
\hline \multicolumn{3}{|l|}{ Therapy } \\
\hline CVC removal (date $\left.{ }^{a}\right)$ & Not applicable & Yes (37) \\
\hline Antifungal therapy after culture & No & No \\
\hline Outcome of patient & Discharge without medical advice & Discharge without medical advice \\
\hline
\end{tabular}

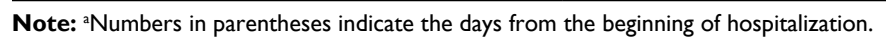

Abbreviation: CVC, central venous catheter. 
(400 mg daily) on Day 28, then her temperature went down to about $38.0^{\circ} \mathrm{C}$. On Day 37 , the central venous catheter (CVC) was removed and sent for culture; meanwhile, central and peripheral blood cultures were also taken. An organism initially identified as Candida albicans/C. tropicalis was recovered from the catheter and blood culture in chromogenic agar, on Day 40. The patient was discharged in a serious condition against medical advice on Day 38 (Table 1).

\section{Materials and methods Identification of $T$. dohaense strains}

The two isolates initially identified as T. asahii and C. albicans/C. tropicalis were isolated from the blood culture and the CVC culture of Patients 1 and 2, respectively (Table 1). The isolates were forwarded to a central laboratory (Department of Clinical Laboratory, Peking Union Medical College Hospital) for identification and further study, as previously described. ${ }^{12}$ At the central laboratory, the two isolates were identified as follows: Patient 1, 10PU193 and Patient 2, 12ZZ130, based on the CHIF-NET system. The two isolates from Patient 2, which were initially identified as $C$. albicans/C. tropicalis, were confirmed as being of $T$. dohaense by rRNA sequencing at the central laboratory, and so was the isolate from Patient 1. The morphological characteristics of these unusual yeast isolates were determined by standard laboratory methods. ${ }^{13}$ In addition, their appearance on chromogenic agar (CHROMagar Candida; CHROMagar Company) was examined and interpreted according to the manufacturer's instructions. To confirm the initial identification results from the referring laboratory, the VITEK 2 YST system was used at the central laboratory.

\section{PCR and sequence-based identification}

Genomic DNA was extracted by beating the suspension of isolates with glass beads, as described earlier. ${ }^{14}$ All the isolates were subsequently identified as $T$. dohaense (see the following section).

\section{Sequence-based identification}

For all the isolates, DNA extraction and amplification of the internal transcribed spacer (ITS) region, the D1/D2 domain of the 26S rRNA gene, and the intergenic spacer region-1 (IGS1) region were performed, respectively, with primer pairs ITS1/ITS4, F63/R635, and 26SF (IGS1-26SS)/5 SR (IGS12-58S), as previously described. ${ }^{15,16}$ The PCR products were sequenced in both directions using the DNA analyzer ABI 3730XL system (Applied Biosystems, Foster City, CA,
USA). Species identification was performed by querying the obtained ITS, D1/D2, and IGS1 sequences against those in the GenBank database, with nucleotide Basic Local Alignment Search Tool (BLASTn, http://blast.ncbi.nlm.nih.gov).

\section{Phylogenetic analysis}

The nucleotide sequences of ITS, D1/D2, and IGS1 regions for type strains of 16 Trichosporon human pathogen species available in GenBank as of 17 April 2018, and additional ITS and D1/D2 regions from literature review (19, 18, and 15 sequences for the ITS region, D1/D2 domain, and IGS1 region, respectively; Figure 3) were downloaded. Phylogenetic analysis was performed with the software Molecular Evolutionary Genetic Analysis (MEGA software, version 6.0; http://www.megasoftware.net) using the neighborjoining (NJ) method, with all positions containing gaps and missing data eliminated from the data set. The significance of the cluster nodes was determined by bootstrapping with 1,000 randomizations, and the NJ Tree was thereafter generated. The ITS, D1/D2, and IGS1 sequences of Cryptococcus neoformans (GenBank accession numbers EU240005, $\mathrm{AF} 075484$, and $\mathrm{KC} 883800$, respectively) were used as outgroups.

\section{Identification and analysis by matrix- assisted laser desorption/ionization time- of-flight mass spectrometry (MALDI-TOF MS)}

The identification of the two $T$. dohaense isolates was further explored using three MALDI-TOF MS systems the bioMérieux Vitek MS (bioMérieux, Marcy l'Etoile, France), Bruker Autoflex Speed (Bruker Daltonics, Bremen, Germany), and an automated mass spectrometry microbial identification system from Autobio Labtec Instruments Co, Ltd (Zhengzhou, China). Samples were prepared as previously described, ${ }^{17}$ and identification of isolates was handled using In Vitro Diagnosis (IVD) Knowledgebase version 2.0 (software Myla, Vitek MS), Biotyper version 3.1 (Bruker Daltonics, Autoflex Speed), and Autof Acquirer version 1.0.26 (Autof MS1000, Autobio), respectively, following manufacturer's criteria for species and genus identification.

The protein profiles or mass spectra of the two isolates were further analyzed by constructing the main spectrum peaks (MSP) dendrogram using the correlation distance measure with the average linkage algorithm setting of the Bruker Biotyper software, ${ }^{17}$ to determine whether their spectra exhibited any differences. 


\section{Antifungal susceptibility testing}

In vitro susceptibility testing of the two $T$. dohaense isolates to fluconazole, voriconazole, itraconazole, posaconazole, caspofungin, micafungin, amphotericin $\mathrm{B}$, and 5-flucytosine was performed by the broth dilution methodology, according to the Clinical and Laboratory Standards Institute document M27-A3 ${ }^{18}$ C. parapsilosis, American Type Culture Collection (ATCC) 22019, and C. krusei, ATCC 6258, were used as quality control strains. It is noteworthy that there are no established breakpoints for Trichosporon species to various antifungal agents, and so it is difficult to precisely confirm its susceptibility to the agents. ${ }^{7,19}$

\section{Literature review}

A PubMed search using the key words "Trichosporon dohaense" was performed, and the identified case studies were reviewed to identify additional information. All the identified papers were carefully read, paying special attention to demographic data, clinical specimens, risk factors, as well as the ITS, D1/D2, and IGS1 sequences of the species.

\section{Nucleotide sequence accession numbers}

The sequences of the ITS region, D1/D2 domain, and IGS1 region have been deposited in GenBank with accession numbers MG857685, MG857767, and MG871991, respectively, for strain 10PU193 (isolated from Patient 1) and accession numbers MG857710, MG857792, and MG872016, respectively, for 12ZZ130 (isolated from Patient 2). These sequences have been deposited as those of strains assigned as T. dohaense.

\section{Ethics statement}

Written informed consent to publish their case details was obtained from the patients or from the next of kin where the patient was unable to provide consent. The Human Research Ethics Committee of Peking Union Medical College Hospital provided permission to publish this report (PUMCHB-B-2-23-1).

\section{Results}

\section{Sequence-based identification}

The two T. dohaense isolates (10PU193 and 12ZZ130) had identical ITS region, D1/D2 domain, and IGS1 region sequences. By querying their sequences against those in the National Center for Biotechnology Information database, the ITS region and the D1/D2 domain sequences of the two T. dohaense strains showed $100 \%$ (405/405 bp) and 100\% (517/517 bp) similarity to the ITS and D1/D2 sequences of T. dohaense type strain CBS10761 ${ }^{\mathrm{T}}$ (GenBank accession number FJ228471 and FJ228475), respectively (Tables 2 and 3). Species identification was not achieved by using the IGS1 region, as there is no T. dohaense reference IGS1 sequence in the GenBank. However, the IGS1 region can clearly distinguish the two isolates from other Trichosporon species (best hit identity $\leq 86 \%$ ).

\section{Phenotypic characteristics of $T$. dohaense}

The two T. dohaense isolates grew well at $28^{\circ} \mathrm{C}$ and $35^{\circ} \mathrm{C}$, but failed to grow at $42^{\circ} \mathrm{C}$, on Sabouraud dextrose agar. The colonies of both isolates were creamy, butyrous, white colored, and slow growing ( $>48$ hours to become a colony of $\sim 1 \mathrm{~mm}$ in diameter on the third zone) on Sabouraud dextrose agar (Figure 1A-D). Microscopic examination showed budding yeast cells, hyphae, and arthroconidia (Figure $1 \mathrm{G}$ and H). On CHROMagar Candida, T. dohaense cells were dark blue, similar to the color of $C$. tropicalis, but the moist colony characteristic was quite different from that of $T$. asahii (Figure 1E and F). In the central laboratory, repeat testing by biochemical identification systems also misidentified the two isolates (10PU193 and 12ZZ130) as T. asahii and T. inkin, respectively, by the VITEK 2 YST (identification rates $85 \%$ and $95 \%$, respectively) system (Table 2 ).

Table 2 Results of identification and antifungal susceptibility testing for Trichosporon dohaense isolates from this study

\begin{tabular}{|c|c|c|}
\hline Patient & $\mathbf{I}$ & 2 \\
\hline Isolate & IOPUI93 & $12 Z Z 130$ \\
\hline \multicolumn{3}{|c|}{ Identification by DNA sequencing } \\
\hline ITS & T. dohaense & T. dohaense \\
\hline DI/D2 & T. dohaense & T. dohaense \\
\hline IGSI & No identification & No identification \\
\hline \multicolumn{3}{|c|}{ Identification by MALDI-TOF MS (scores/confidence values ${ }^{\mathrm{a}}$ ) } \\
\hline Bruker Biotyper & T. dohaense (2.193) & T. dohaense (2.196) \\
\hline \multirow[t]{2}{*}{ Vitek MS } & T. ovoides $(50) /$ & T. ovoides (99.9) \\
\hline & T. asteroides (50) & \\
\hline Autobio MS & T. dohaense $(9.506)$ & T. dohaense $(9.562)$ \\
\hline VITEK 2 YST (rate) & T. asahii $(85 \%)$ & T. inkin (95\%) \\
\hline \multicolumn{3}{|c|}{ Antifungal susceptibility by broth microdilution $(\mu \mathrm{g} / \mathrm{mL})$} \\
\hline Caspofungin & $>8$ & $>8$ \\
\hline Micafungin & $>8$ & $>8$ \\
\hline Fluconazole & 8 & 16 \\
\hline Voriconazole & 0.25 & I \\
\hline Posaconazole & 0.25 & 0.5 \\
\hline Itraconazole & 0.5 & 0.5 \\
\hline Amphotericin B & 1 & 1 \\
\hline 5-Flucytosine & 4 & 4 \\
\hline
\end{tabular}

Notes: aScores for Bruker Biotyper, Vitek MS, and Autobio MS vary within the ranges of $0-3,0-100$, and $0-10$, with zero indicating no relationship and the maximum indicating identical spectrum profiles.

Abbreviations: ITS, internal transcribed spacer; MALDI-TOF-MS, matrix-assisted laser desorption/ionization time-of-flight mass spectrometry; IGS-I, intergenic spacer region-I. 


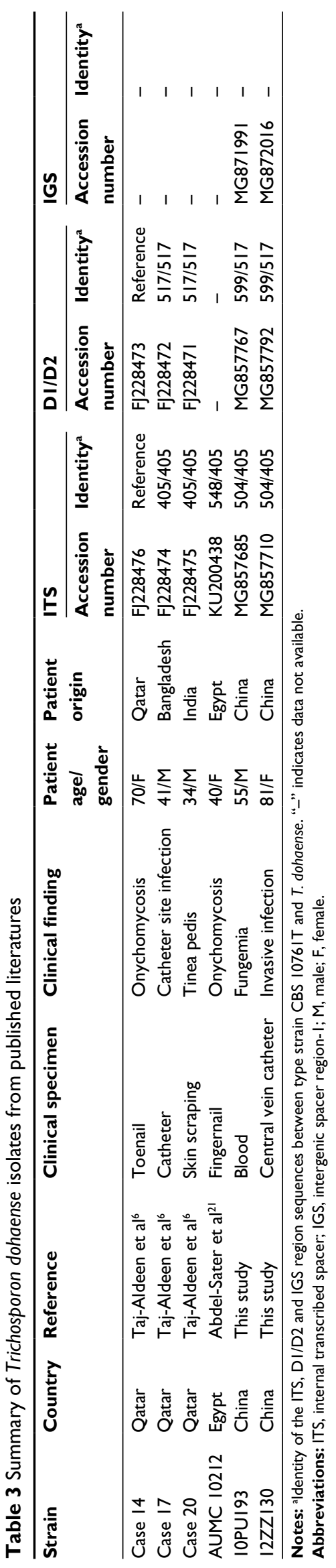

\section{Phylogenetic analysis}

To investigate the genetic relatedness of the two T. dohaense strains, the ITS, D1/D2, and IGS1 sequences of T. dohaense isolated from this study and those of 16 Trichosporon species with clinical relevance available in GenBank were subjected to phylogenetic analysis (Figure 3). By NJ analysis, all the ITS, D1/D2, and IGS1 sequence alignments clustered the two T. dohaense isolates with T. dohaense type strain CBS10761 ${ }^{\mathrm{T}}$, thus clearly distinguishing them from other Trichosporon species recovered from humans (Figure 3). Based on the ITS, D1/D2, and IGS1 sequences, the 16 Trichosporon species with clinical relevance identified in the literature clustered in three clades, including Ovoides, Gracile/Brassicae, and Cutaneum. Moreover, T. dohaense belongs to the Ovoides clade (Figure 3). In addition, the IGS1 region showed the highest variation among the three regions, and the $T$. dohaense cluster corresponded to a unique species, with the IGS1 region of T. dohaense ( $824 \mathrm{bp}$ ) being much longer than that of other species (195-704 bp).

\section{MALDI-TOF MS analysis}

The MS spectra of T. dohaense were not covered in the Vitek MS database but were available in the Bruker Biotyper and Autobio MS identification databases. Hence, the Vitek MS system misidentified the two isolates as T. ovoides/T. asteroids and T. ovoides, with confidence values of 50/50 and 99.9, respectively, whereas Bruker Biotyper and Autobio MS correctly identified the isolates (Table 2). In addition, the Bruker Biotyper system MSP dendrogram revealed that the spectra of the two isolates were identical (Figure 2). At a distance-level cutoff of 900, 19 Trichosporon species were divided into three clusters, which had basic consistency with the taxon of the clades on analysis by rRNA gene tools, except for T. montevideense and T. mycotoxinivorans. Our two isolates clustered with the $T$. dohaense isolates in the database and were clearly distinct from other species, as shown by the MSP dendrogram.

\section{Antifungal susceptibilities}

Table 2 shows that both $T$. dohaense isolates in the present study needed high minimal inhibitory concentrations (MICs) of caspofungin and micafungin, which was considered to be natural resistance to these agents (MIC $>8 \mu \mathrm{g} / \mathrm{mL}$ ). ${ }^{20}$ The two isolates had intermediate susceptibility to itraconazole (MIC $0.5 \mu \mathrm{g} / \mathrm{mL}$ ), voriconazole (MIC 0.25 and $1 \mu \mathrm{g} / \mathrm{mL}$ ), posaconazole (MIC 0.25 and $0.5 \mu \mathrm{g} / \mathrm{mL}$ ), and fluconazole (MIC 8 and $16 \mu \mathrm{g} / \mathrm{mL}$ ). The MICs for 5-flucytosine and amphotericin B were 4 and $1 \mu \mathrm{g} / \mathrm{mL}$, respectively. 


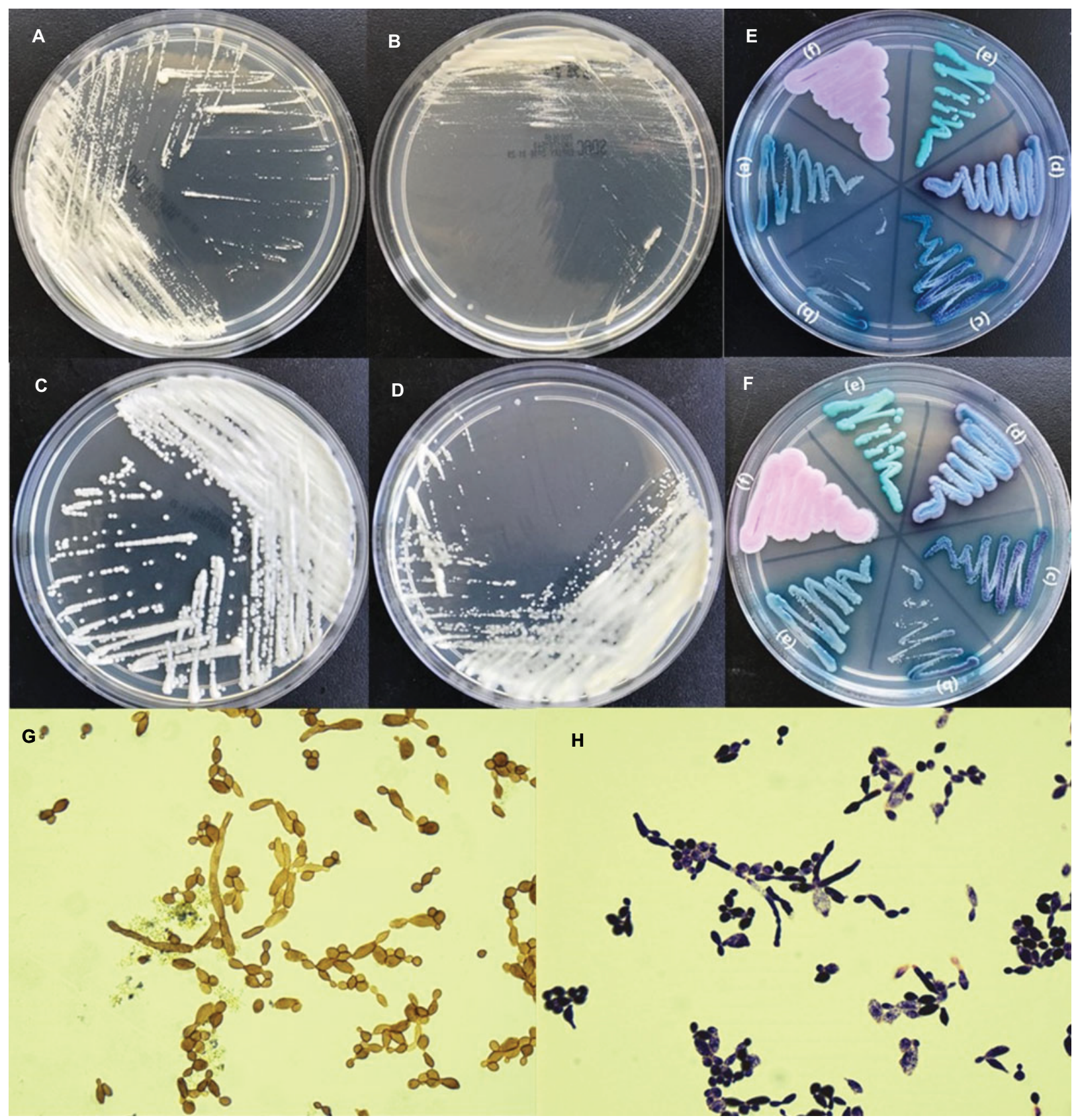

Figure I Phenotypic characteristics of two Trichosporon dohaense isolates.

Notes: The isolates were grown on Sabouraud dextrose agar (A-D), chromogenic media CHROMagar Candida (E, F) and budding yeast cells, hyphae, and arthroconidia on Sabouraud dextrose agar by periodic acid methenamine silver staining and Gram's staining, respectively $(\mathbf{G}, \mathbf{H})$ : Incubation conditions: $\mathbf{A}, \mathbf{B}$, and $\mathbf{E}-37^{\circ} \mathrm{C}, 48$ hours; $\mathbf{C}$, D, and F-H $-37^{\circ} \mathrm{C}, 72$ hours; A and C, T. dohaense IOPUI93; B and D, T. dohaense I2ZZI30. In E and F, (a) indicates T. dohaense IOPUI93; (b) T. dohaense I2ZZI30; (c) T. asahii CBS 2479; (d) C. tropicalis 10HI048; (e) C. albicans ATCC 90028; and (f) C. krusei ATCC 6258. The (d) isolate in panels E and F was selected from the CHIF-NET study. ${ }^{12}$

Abbreviation: CHIF-NET, China Hospital Invasive Fungal Surveillance Net.

\section{Literature review}

Only two relevant articles on T. dohaense were identified through PubMed literature search, with the reported strains all isolated from superficial infections. Three T. dohaense cases, including one case each of tinea pedis, onychomycosis, and superficial catheter site, were first described in Doha, Qatar, by Taj-Aldeen et al. ${ }^{6}$ After this original description, the second case of $T$. dohaense was reported from a case of onychomycosis in 2016 in Assiut, Egypt. ${ }^{21}$ We concluded that, in the first of the two articles identified in the literature, an immunocompromised state was the risk factor for infection by T. dohaense. Both the ITS and the D1/D2 sequences of $T$. dohaense strains in the previous studies clustered with those of the two isolates in our study, but only the ITS 


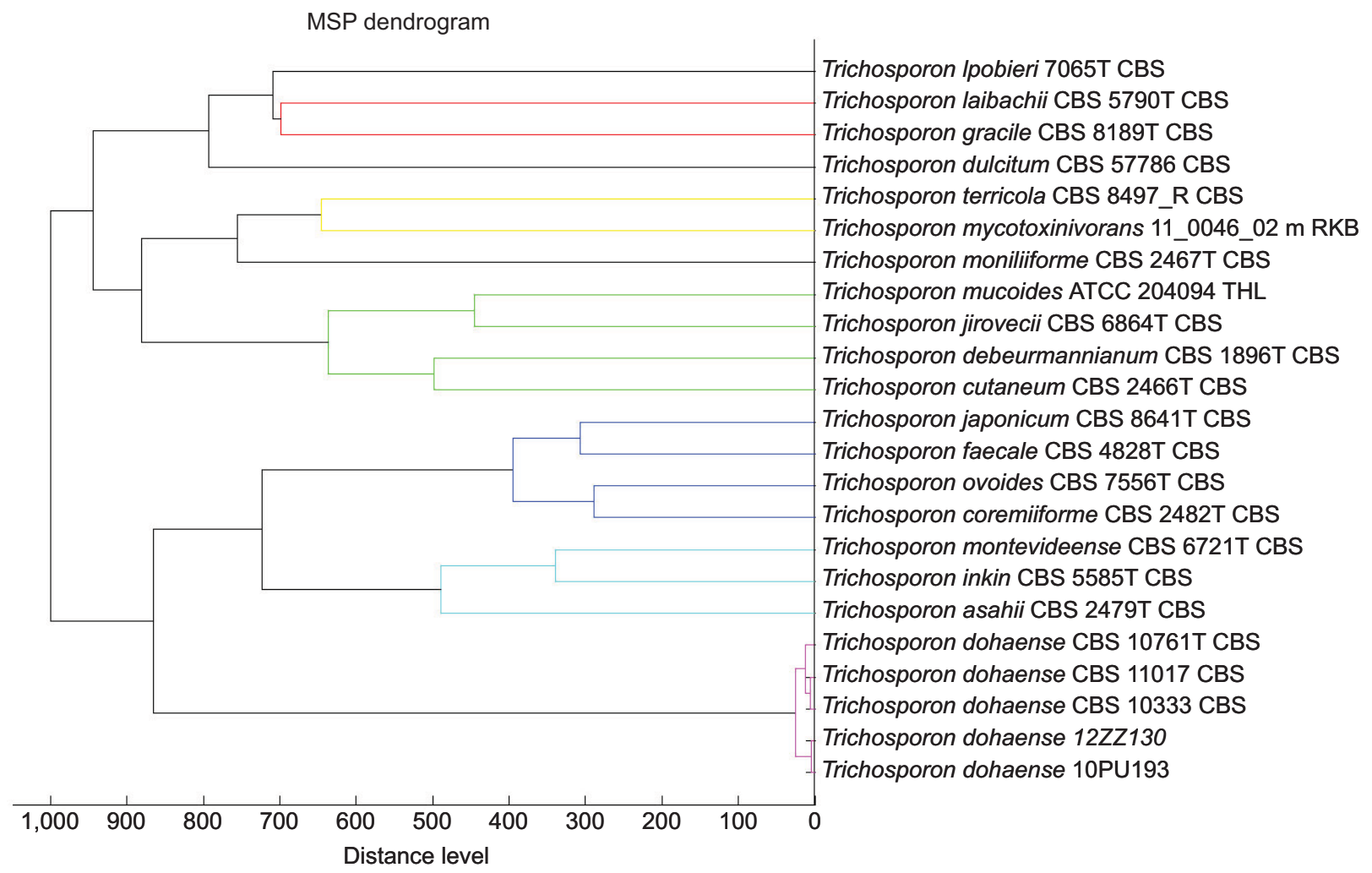

Figure 2 The MSP dendrogram generated from the protein mass spectra of the two Trichosporon dohaense isolates studied by Bruker Biotyper (Bremen, Germany), version 3.1.

Abbreviation: MSP, main spectrum peaks.

sequence was mentioned by the second report. The MSP dendrogram generated from the protein mass spectra of T. dohaense isolates from the first report also clustered with our two isolates (Table 3, Figures 2 and 3). ${ }^{6,21}$ Unfortunately, there was no IGS1 sequence available from literature review for investigation of the regional divergence of the species.

\section{Discussion}

T. dohaense strains are rarely identified in the clinical microbiology laboratory, and the pathogenic role of this species remains uncertain. To the best of our knowledge, this is the first report on invasive infections caused by this rare Trichosporon species.

By detailed retrospective review of the medical chart records, we discovered that both the patients presenting with $T$. dohaense invasive infection suffered from tumor and underlying diseases. Cancer, vascular and urinary catheters, organ transplantation, and broad-spectrum antibiotic therapy are considered the main risk factors for invasive trichosporonosis. Both patients were in an immunocompromised state and had classical risk factors for invasive trichosporonosis; ; ${ }^{7,22}$ both were on broad-spectrum antibiotic therapy and ICU hospitalization. Patient 1 had undergone chemotherapy and hemodialysis, and Patient 2 had undergone major surgery within 30 days, had central venous catheters in situ, and was receiving parenteral nutrition. The risk factors for $T$. dohaense invasive infections are similar to those for other Trichosporon species. ${ }^{8,23}$

Both patients were in a serious condition but got discharged against medical advice and were subsequently lost to follow-up. Although failure to follow up the patient to document prognosis and response to antifungal therapy is a limitation of our study, the course of infection in the two patients during hospitalization suggests a genuine infection rather than contamination or colonization with $T$. dohaense. Both patients were immunocompromised as they were cancer sufferers who accepted chemotherapy and thus were more susceptible to fungal infection. T. dohaense was recovered from the culture of blood from Patient 1. Furthermore, the patient had fever, leukocytosis, and elevated C-reactive protein, which is consistent with a fungal bloodstream infection. ${ }^{24}$ The two isolates recovered from the blood culture from Patient 1 and the CVC culture from Patient 2 were both identified as $T$. dohaense and met the standard guidelines of catheter-associated bloodstream infections. ${ }^{25}$ Patient 2 had a continuous fever for 10 days and the temperature decreased 


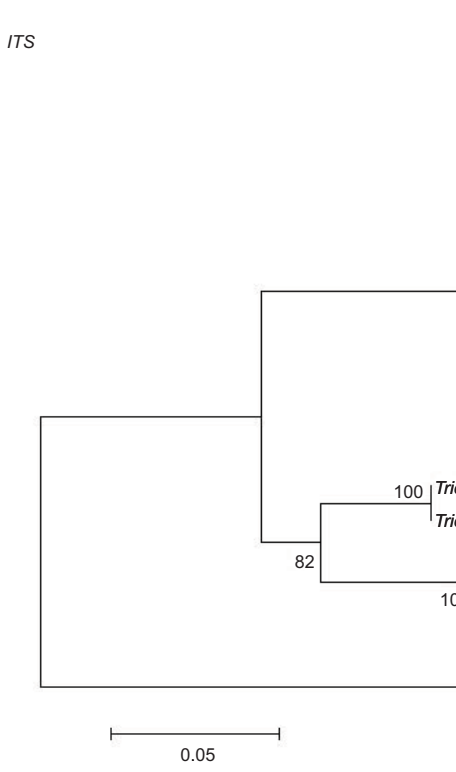

62 Trichosporon ovoides CBS 7556/NR 073254.1

52 Lrichosporon inkin CBS 5585/NR 073243.1

20 - Trichosporon ilactis CBS 9051/KY105749.

18 Trichosporon faecale CBS 4828/AF444419.1

32 [ Trichosporon asteroides CBS 2481/AF444416.1

5676 Trichosporon japonicum CBS 8641/AF444473.1

56 Trichosporon coremiiforme CBS 2482/AF444434.1

Trichosporon asahii CBS 2479/FJ943429.1

100

Thosporon dohaense 10PU 193/MG857685

Trichosporon dohaense 12ZZ130/MG857710

Trichosporon dohaense AUMC 10212/KU200438.1

99 Trichosporon dohaense CBS 10761/FJ228475.1

Trichosporon dohaense CBS 10333/FJ228474.1

Trichosporon dohaense CBS 11017/FJ228476.1

Trichosporon montevideense CBS 6721/JN943734.1

Trichosporon domesticum CBS 8280/JN943742.1

- Cutaneotrichosporon cutaneum CBS 2466/NR 073216.1

- Cutaneotrichosporon mucoides CBS 7625/NR 073246.1

83 [ Cutaneotrichosporon jirovecii CBS 6864/AF 444437.1

${ }_{51}$ Cutaneotrichosporon dermatis CBS 2013/NR 130667.1
Ovoides

Gracile/brassicae

Cutaneum
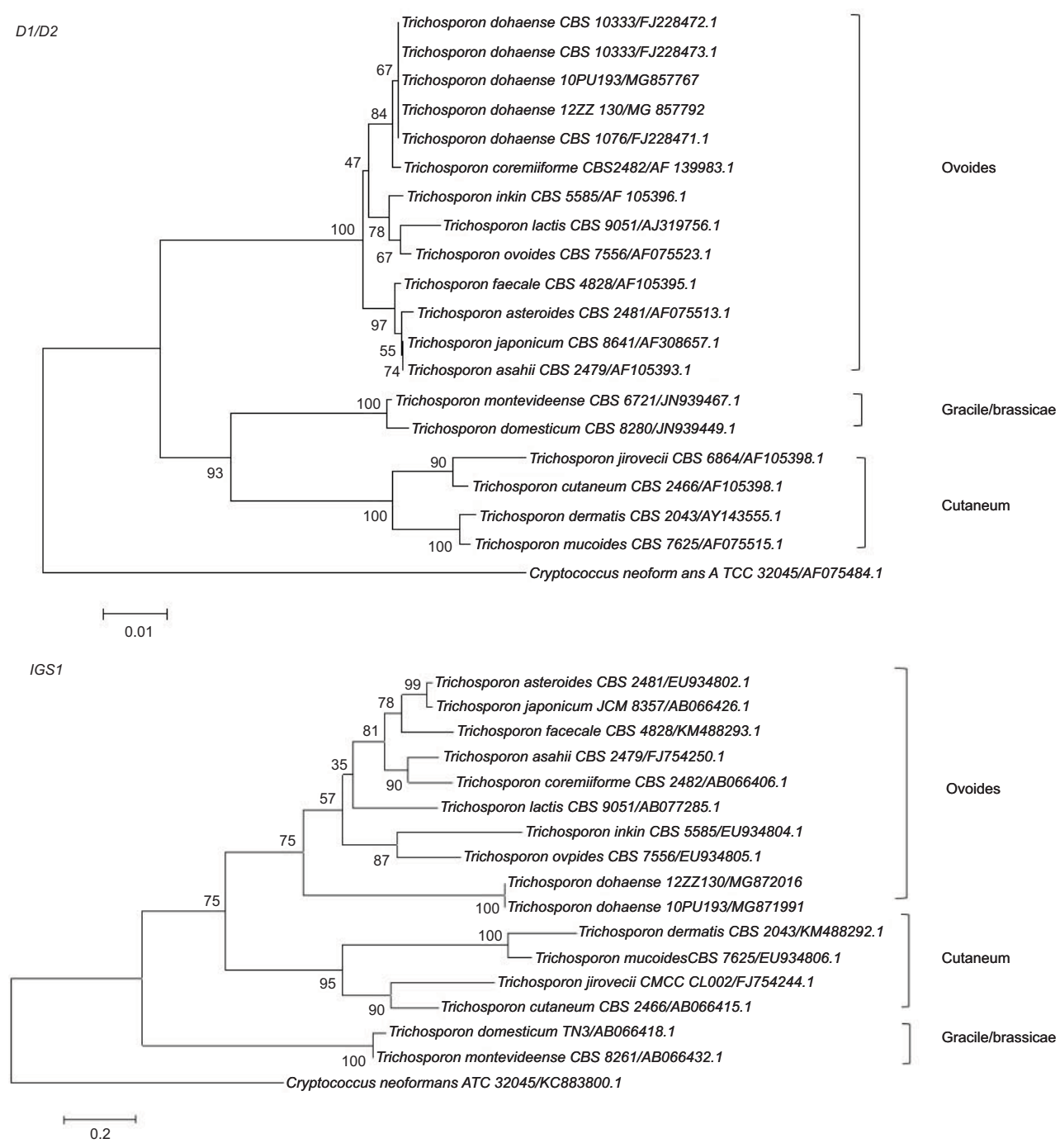

Figure 3 The $\mathrm{NJ}$ tree of Trichosporon dohaense generated from T. dohaense ITS, DI/D2, and IGSI sequences from this study and the I6 Trichosporon species with clinical relevance available in GenBank, with Cryptococcus neoformans (GenBank accession number EU240005, AF075484, and KC883800, respectively) used as the outgroup. Abbreviations: IGS, intergenic spacer region; ITS, internal transcribed spacer; NJ, neighbor-joining. 
from $39.2^{\circ} \mathrm{C}$ to $38^{\circ} \mathrm{C}$ after fluconazole therapy. An abundant cell wall polysaccharide, $(1,3)-\beta$-D-glucan, is found in most fungi and is important in the diagnosis of invasive fungal infections. Patient 2 had a significantly raised level of this polysaccharide in the blood. Both patients had negative bacterial cultures and showed no response to antibiotic treatment. Based on the evidence outlined herein, it appears highly likely that the two T. dohaense strains isolated from the two patients were pathogens.

Misidentification of the T. dohaense isolates (10PU193 and 12ZZ130) was noted during our study, with the species easily confused with $C$. tropicalis and C. albicans on CHROM agar, respectively. Furthermore, a commonly used phenotypic identification system VITEK 2 YST may misidentify the species as two commonly encountered Trichosporon species, T. asahii and T. inkin. Accurate identification of $T$. dohaense is important to avoid administration of inappropriate antifungal therapy. DNA-based methods have been extensively used for the accurate identification of Trichosporon spp. Although the ITS region and the D1/D2 domain sequences were able to correctly assign the two $T$. dohaense isolates to species level, the IGS1 region is considered more suitable for accurate species identification of Trichosporon due to the higher discriminatory power. ${ }^{26}$ Of note, there is no IGS1 region sequence available in the GenBank database, and our data provide the basis for future research.

The recent development of MALDI-TOF MS has provided a promising alternative for routine identification of Trichosporon species. ${ }^{27,28}$ The mass spectral data of T. dohaense were not represented in Vitek MS but in Bruker Biotyper and Autobio MS databases. Bruker Biotyper and Automated MS have the capacity to accurately identify T. dohaense, whereas the Vitek MS systems does not.

To this end, the mass spectra of the two T. dohaense strains in our study and the 19 Trichosporon species described in other studies were archived into the customer-specific sublibrary of the Biotyper software, following the manufacturer's protocol. Our analysis of the MSP dendrogram generated by the Bruker Biotype system showed that the spectra of the two clinical isolates described in this study clustered with the spectra of $T$. dohaense provided by the system. Furthermore, the protein heterogeneity clearly separated the $T$. dohaense isolates from other species, which was in accordance with the rRNA-discriminated clades, except for two species. Phylogenetic analysis demonstrated that the 16 Trichosporon species were clustered by rRNA sequences to Ovoides, Gracile/Brassicae, and Cutaneum clades, with $T$. dohaense confined to the Ovoides clade, which is consistent with results from previous studies. ${ }^{7,10}$ Although the ITS, D1/D2, and IGS1 regions can all clearly discriminate $T$. dohaense from other Trichosporon species, a comparative analysis of the phylogenetic tree constructed in our study suggested that the IGS1 region has the highest variation among the three regions.

Trichosporon has been reported to be the most common cause of noncandidal yeast infections in patients with hematological malignancies, and the infections are related to high mortality rates. ${ }^{29}$ Since different Trichosporon species may exhibit different antifungal susceptibilities, ${ }^{7}$ accurate identification is important to avoid inappropriate antifungal treatment. In our study, micafungin and caspofungin demonstrated no in vitro activity against $T$. dohaense as with other Trichosporon species. ${ }^{3,4} T$. dohaense demonstrated the highest susceptibility to amphotericin B, with MIC of $1 \mu \mathrm{g} /$ $\mathrm{mL}$, which is in agreement with the findings of Taj-Aldeen et al. ${ }^{6}$ We also noted that the MICs of the four azoles, especially fluconazole ( 8 and $16 \mu \mathrm{g} / \mathrm{mL}$ ), for our two isolates are apparently higher than those from a previous study (1-4 $\mu \mathrm{g} / \mathrm{mL}) .{ }^{6}$ Furthermore, the MICs of the four azoles in our study were also higher than the $\mathrm{MIC}_{50}$ value of 43 isolates of Trichosporon species isolated from Taiwan during an 8-year study period with MICs of $4 \mu \mathrm{g} / \mathrm{mL}$ for fluconazole, 0.03 $\mu \mathrm{g} / \mathrm{mL}$ for voriconazole, $0.12 \mu \mathrm{g} / \mathrm{mL}$ each for itraconazole and posaconazole, and $0.5 \mu \mathrm{g} / \mathrm{mL}$ for amphotericin B. ${ }^{22}$ And the MICs of fluconazole and voriconazole for our study isolates was also higher than the MIC geomean of 62 T. asahii isolates for the CHIF-NET program (fluconazole $4 \mu \mathrm{g} / \mathrm{mL}$, voriconazole $0.064 \mu \mathrm{g} / \mathrm{mL}$ ). However, the current clinical data were insufficient to predict the correlation between in vitro susceptibility and in vivo response to therapy since the patients had not received antifungal therapy after culture.

\section{Conclusion}

This study points to a possible emergence of $T$. dohaense as a significant opportunistic human invasive pathogen. The high MICs to all echinocandins and reduced susceptibility to azoles highlight the importance of accurate identification for appropriate therapy. Molecular methods are needed for accurate identification and further molecular characterization of this fungal species, in addition to conventional laboratory tests.

\section{Acknowledgments}

This work was supported by the Special Research Foundation for Capital Medical Development: Improvement of 
laboratory surveillance system and establishment of diagnostic service mode for invasive fungal diseases in Beijing (grant number 2016-1-4013), Chinese Academy of Medical Sciences (CAMS) Innovation Fund for Medical Sciences (CIFMS) (grant number 2016-I2M-1-014), and the Youth Research Foundation in Peking Union Medical College: Novel Identification Assays of Clinical Important Invasive Fungal Species (grant number 2012X03).

\section{Author contributions}

S-YY, L-NG, and Y-CX designed the studies. S-YY, J-WC, and M-LZ performed the phenotypic assays, PCR and sequence-based identification, and antifungal susceptibility testing. S-YY and HW carried out phylogenetic analysis and literature review. HX performed the case analysis. S-YY wrote the manuscript with contributions from all authors. MX, FK, and TK revised the manuscript. All authors contributed toward data analysis, drafting and critically revising the paper and agree to be accountable for all aspects of the work.

\section{Disclosure}

The authors report no conflicts of interest in this work.

\section{References}

1. Sugita T, Ikeda R, Nishikawa A. Analysis of Trichosporon isolates obtained from the houses of patients with summer-type hypersensitivity pneumonitis. J Clin Microbiol. 2004;42(12):5467-5471.

2. Liao Y, Lu X, Yang S, Luo Y, Chen Q, Yang R. Epidemiology and outcome of trichosporon fungemia: a review of 185 reported cases from 1975 to 2014. Open Forum Infect Dis. 2015;2(4):ofv141.

3. Montoya AM, Sánchez González A, Palma-Nicolás JP, Gómez-Treviño A, González JG, González GM. Genotyping, extracellular compounds, and antifungal susceptibility testing of Trichosporon asahii isolated from Mexican patients. Med Mycol. 2015;53(5):505-511.

4. Guo LN, Xiao M, Kong F, et al. Three-locus identification, genotyping, and antifungal susceptibilities of medically important Trichosporon species from China. J Clin Microbiol. 2011;49(11):3805-3811.

5. Mariné M, Brown NA, Riaño-Pachón DM, Goldman GH. On and under the skin: emerging basidiomycetous yeast infections caused by Trichosporon species. PLoS Pathog. 2015;11(7):e1004982.

6. Taj-Aldeen SJ, Al-Ansari N, El Shafei S, et al. Molecular identification and susceptibility of Trichosporon species isolated from clinical specimens in Qatar: isolation of Trichosporon dohaense Taj-Aldeen, Meis \& Boekhout sp. nov. J Clin Microbiol. 2009;47(6):1791-1799.

7. Colombo AL, Padovan AC, Chaves GM. Current knowledge of Trichosporon spp. and Trichosporonosis. Clin Microbiol Rev. 2011;24(4):682-700.

8. de Almeida Júnior JN, Hennequin C. Invasive Trichosporon infection: a systematic review on a re-emerging fungal pathogen. Front Microbiol. 2016;7:1629.

9. Chagas-Neto TC, Chaves GM, Melo AS, Colombo AL. Bloodstream infections due to Trichosporon spp.: species distribution, Trichosporon asahii genotypes determined on the basis of ribosomal DNA intergenic spacer 1 sequencing, and antifungal susceptibility testing. J Clin Microbiol. 2009;47(4):1074-1081.
10. Taverna CG, Córdoba S, Murisengo OA, Vivot W, Davel G, BoscoBorgeat ME. Molecular identification, genotyping, and antifungal susceptibility testing of clinically relevant Trichosporon species from Argentina. Med Mycol. 2014;52(4):356-366.

11. Hickey PW, Sutton DA, Fothergill AW, et al. Trichosporon mycotoxinivorans, a novel respiratory pathogen in patients with cystic fibrosis. $J$ Clin Microbiol. 2009;47(10):3091-3097.

12. Wang $\mathrm{H}$, Xiao M, Chen SC, et al. In vitro susceptibilities of yeast species to fluconazole and voriconazole as determined by the 2010 National China Hospital Invasive Fungal Surveillance Net (CHIF-NET) study. J Clin Microbiol. 2012;50(12):3952-3959.

13. Li J, Xu YC, Bai FY. Candida pseudorugosa sp. nov., a novel yeast species from sputum. J Clin Microbiol. 2006;44(12):4486-4490.

14. Amberg DC, Burke DJ, Strathern JN. Methods in Yeast Genetics: A Cold Spring Harbor Laboratory Course Manual. New York: Cold Spring Harbor Laboratory Press; 2005.

15. Sugita T, Nakajima M, Ikeda R, Matsushima T, Shinoda T. Sequence analysis of the ribosomal DNA intergenic spacer 1 regions of Trichosporon species. J Clin Microbiol. 2002;40(5):1826-1830.

16. Diaz MR, Fell JW. High-throughput detection of pathogenic yeasts of the genus trichosporon. J Clin Microbiol. 2004;42(8):3696-3706.

17. Pulcrano G, Roscetto E, Iula VD, Panellis D, Rossano F, Catania MR. MALDI-TOF mass spectrometry and microsatellite markers to evaluate Candida parapsilosis transmission in neonatal intensive care units. Eur J Clin Microbiol Infect Dis. 2012;31(11):2919-2928.

18. Clinical and Laboratory Standards Institute. Reference method for broth dilution antifungal susceptibility testing of yeasts. Approved standard 3rd ed. CLSI document M27-A3. Wayne, PA: Humana Press; 2008.

19. Rastogi V, Honnavar P, Rudramurthy SM, Pamidi U, Ghosh A, Chakrabarti A. Molecular characterisation and antifungal susceptibility of clinical Trichosporon isolates in India. Mycoses. 2016;59(8):528-534.

20. Clinical and Laboratory Standards Institute. Reference method for broth dilution antifungal susceptibility testing of yeasts. Fourth informational supplement M27-S4. Wayne, PA: Clinical and Laboratory Standards Institute; 2012.

21. Abdel-Sater MA, Moubasher AA, Soliman Z. Identification of three yeast species using the conventional and internal transcribed spacer region sequencing methods as first or second global record from human superficial infections. Mycoses. 2016;59(10):652-661.

22. Ruan SY, Chien JY, Hsueh PR. Invasive trichosporonosis caused by Trichosporon asahii and other unusual Trichosporon species at a medical center in Taiwan. Clin Infect Dis. 2009;49(1):e11-e17.

23. Yamamoto M, Takakura S, Hotta G, et al. Clinical characteristics and risk factors of non-Candida fungaemia. BMC Infect Dis. 2013;13:247.

24. Martinez RM, Wolk DM. Bloodstream Infections. Microbiol Spectr. 2016;4(4).

25. Zakhour R, Chaftari AM, Raad II. Catheter-related infections in patients with haematological malignancies: novel preventive and therapeutic strategies. Lancet Infect Dis. 2016;16(11):e241-e250.

26. Kalkanci A, Sugita T, Arikan S, et al. Molecular identification, genotyping, and drug susceptibility of the basidiomycetous yeast pathogen Trichosporon isolated from Turkish patients. Med Mycol. 2010;48(1):141-146.

27. Kolecka A, Khayhan K, Groenewald M, et al. Identification of medically relevant species of arthroconidial yeasts by use of matrix-assisted laser desorption ionization-time of flight mass spectrometry. J Clin Microbiol. 2013;51(8):2491-2500.

28. de Almeida Júnior JN, Figueiredo DS, Toubas D, et al. Usefulness of matrix-assisted laser desorption ionisation-time-of-flight mass spectrometry for identifying clinical Trichosporon isolates. Clin Microbiol Infect. 2014;20(8):784-790.

29. Pfaller MA, Diekema DJ. Rare and emerging opportunistic fungal pathogens: concern for resistance beyond Candida albicans and Aspergillus fumigatus. J Clin Microbiol. 2004;42(10):4419-4431. 
Infection and Drug Resistance is an international, peer-reviewed openaccess journal that focuses on the optimal treatment of infection (bacterial, fungal and viral) and the development and institution of preventive strategies to minimize the development and spread of resistance. The journal is specifically concerned with the epidemiology of antibiotic resistance and the mechanisms of resistance development and diffusion in both hospitals and the community. The manuscript management system is completely online and includes a very quick and fair peerreview system, which is all easy to use. Visit http://www.dovepress.com/ testimonials.php to read real quotes from published authors.

Submit your manuscript here: https://www.dovepress.com/infection-and-drug-resistance-journal 